\section{What Rheumatologists Should Know About Gout and Cardiovascular Disease}

\section{To the Editor:}

We read with interest the recent article by Neogi on asymptomatic hyperuricemia $^{1}$

Currently there is a great deal of interest about the link between serum uric acid (SUA) levels and cardiovascular events. In particular, it is still a matter of debate whether SUA is a predictor or even a causative factor of cardiovascular diseases (CVD).

After the first observation and the inconsistent data of a number of subsequent studies, recent evidence points to hyperuricemia as a risk factor for CVD or even as an independent predictor of mortality at least in individuals at high CVD risk, such as those with preexisting cardiovascular disease, diabetic patients, stroke survivors, or hypertensive patients ${ }^{2}$. Moreover, the risk of CVD as well as of death from all causes seems particularly high for male patients with severe gout ${ }^{3}$.

In recent years, also among rheumatologists, there has been considerable interest in the metabolic syndrome, a clustering of vascular risk factors that promote the development of atherosclerotic CVD; epidemiologic studies, in fact, indicated that the prevalence of metabolic syndrome significantly increases with increasing $\mathrm{SUA}^{4}$, and several clinical and biochemical abnormalities of metabolic syndrome, including insulin resistance, obesity, and hyperlipidemia, are frequently found in gouty patients. In particular, insulin resistance, which seems to play a major role in metabolic syndrome, is probably also the major cause of high SUA in patients with metabolic syndrome, with the compensatory hyperinsulinemia the likely cause of the decreased renal clearance of uric acid. Further, abdominal obesity, one of the metabolic syndrome pathophysiologic abnormalities, has been found to be significantly related to SUA levels, probably because obesity interferes with urate synthesis and excretion.

The relationship between SUA and metabolic syndrome-related CVD risk is further complicated, not only because gout or increased SUA levels are typically seen in middle-aged, overweight patients with hypertension and hyperlipidemia, but also by the recent observation that elevated SUA levels represent an increased CVD risk independent from the coexistence of metabolic syndrome ${ }^{5}$.

Despite these controversies, among the 10 key recommendations developed by the European League Against Rheumatism Task Force regarding gout, particular attention has been paid to associated comorbidities and metabolic syndrome ["risk factors for gout and associated comorbidity should be assessed, including features of metabolic syndrome (obesity, hyperglycemia, hyperlipidemia, hypertension)]" ${ }^{\prime 6}$, and Lin, et al recently suggested that hyperuricemia should be treated as a major metabolic abnormality and included in the definition of metabolic syndrome ${ }^{7}$.

However, the precise role played by SUA in CVD and the underlying mechanism(s) are still undefined, although several mechanisms, including oxidative stress, inflammation, endothelial dysfunction and platelet adhesiveness may be involved.

Further studies are needed first to understand whether SUA is an active component of metabolic syndrome or an "innocent bystander," with the aim to clarify either the usefulness of routine measurement of SUA in patients at high risk of CVD or the role of therapies that lower SUA.

Although the recommended initial treatment of metabolic syndrome is based on lifestyle interventions, with particular attention to weight reduc- tion $^{8}$, it seems reasonable that reduction of SUA would be beneficial at least in high risk patients. The present evidence does not recommend hypouricemic therapy for cardioprotection ${ }^{9}$, but high risk patients such as those with coronary heart disease with SUA $>10 \mathrm{mg} / \mathrm{dl}$ should probably receive hypouricemic drugs ${ }^{10}$, and an aggressive control of cardiovascular risk factors must be performed in patients with gout ${ }^{6}$.

The role of SUA in CVD remains undefined. Since uric acid assays are clinically available and inexpensive, large interventional studies are now needed to clarify if there are patients who may benefit from its measurement, and to assess whether lowering serum urate will impact positively on morbidity and mortality in high-risk patients.

BIANCA MARASINI, MD, Associate Professor; MARCO MASSAROTTI, MD, Rheumatology Unit, IRCCS Istituto Clinico Humanitas, University of Milan, Via Manzoni 56, 20089 Rozzano, Milan, Italy. Address reprint requests to Dr. Massarotti.

\section{REFERENCES}

1. Neogi T. Asymptomatic hyperuricemia: Perhaps not so benign? [editorial]. J Rheumatol 2008;35:734-7.

2. Ioachimescu AG, Brennan DM, Hoar BM, Hazen SL, Hoogwerf BJ. Serum uric acid is an independent predictor of all-cause mortality in patients at high risk of cardiovascular disease: a preventive cardiology information system (PreCIS) database cohort study. Arthritis Rheum 2008;58:623-30.

3. Choi HK, Curhan G. Independent impact of gout on mortality and risk for coronary heart disease. Circulation 2007;116:894-900.

4. Tsouli SG, Liberopoulos EN, Mikhailidis DP, Athyros VG, Elisaf MS. Elevated serum uric acid levels in metabolic syndrome: an active component or an innocent bystander? Metabolism 2006;55:1293-301.

5. Hjortnaes J, Algra A, Olijhoek J, et al. Serum uric acid levels and risk for vascular diseases in patients with metabolic syndrome. J Rheumatol 2007;34:1882-7.

6. Zhang W, Doherty M, Pascual E, et al. EULAR evidence based recommendations for gout. Part I: Diagnosis. Report of a task force of the Standing Committee for International Clinical Studies Including Therapeutics (ESCISIT). Ann Rheum Dis 2006;65:1301-11.

7. Lin WY, Liu CS, Li TC, Cren CC, Li CI, Lin CC. In addition to insulin resistance and obesity, hyperuricemia is strongly associated with metabolic syndrome using different definitions in Chinese populations: a population-based study (Taichung Community Health Study). Ann Rheum Dis 2008;67:432-3.

8. Giugliano D, Ceriello A, Esposito K. Are there specific treatments for the metabolic syndrome? Am J Clin Nutr 2008;87:8-11.

9. Baker JF, Krishnan E, Chen L, Schumacher HR. Serum uric acid and cardiovascular disease: recent developments, and where do they leave us? Am J Med 2005;118:816-26.

10. Strazzullo P, Puig JG. Uric acid and oxidative stress: relative impact on cardiovascular risk? Nutr Metab Cardiovasc Dis 2007;17:409-17.

J Rheumatol 2009;36:4;doi:10.3899/jrheum.081039 